\title{
Topological Defects in an Open Universe
}

\author{
D.N. Spergel and U. Pen ${ }^{a *}$

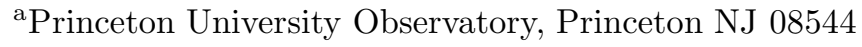

This talk will explore the evolution of topological defects in an open universe. The rapid expansion of the universe in an open model slows defects and suppresses the generation of CBR fluctuations at large angular scale as does the altered relationship between angle and length in an open universe. Defect models, when normalized to COBE in an open universe, predict a galaxy power spectrum consistent with the galaxy power spectrum inferred from the galaxy surveys and do not require an extreme bias. Neither defect models in a flat universe nor standard inflationary models can fit either the multipole spectrum or the power spectrum inferred from galaxy surveys.

\section{ORIGIN OF DENSITY FLUCTUA- TIONS}

The big bang theory, despite its successes in explaining the Hubble expansion, the thermal cosmic background radiation (CBR) and light element nucleosynthesis, is incomplete. For the big bang theory, the notion that the geometry and evolution of the universe is described by the Friedman-Robertson-Walker (FRW) metric, does not explain the origin of the density fluctuations that grew to form today's galaxies and large scale structure.

There are two classes of extensions of the standard big bang model: either the universe began the FRW phase with density variations on scales that were then superhorizon or the universe began the FRW phase without fluctuations and subsequent causal physics generated density fluctuations. Models with primordial potential fluctuations can be classified as "curvature models" as they begin the FRW phase with variations in the space curvature. The generation of fluctuations in an initially smooth or "isocurvature" universe requires variations in the equation of state. Inflationary theories fall into the former category as they produce curvature fluctuations during a deSitter phase that predated the FRW phase. Models with topological defects fall into the latter category as strings or textures generate density fluc-

\footnotetext{
*Research supported by NSF AST88-58145 (PYI), AST90-20863, NASA NAGW-2448 (ATP) and the NSF Grand Challenge Cosmology Consortium
}

tuations in initially smooth universes.

There are a variety of physical mechanism that can lead to spatial variations in the equation of state. Peebles proposed that there are spatial variations in the ratio of baryons to photons. Since baryons and photons have different equations of state, these entropy fluctuations produce density fluctuations [1]. If there was a largescale magnetic field, then its dynamics would lead to large-scale variations in the equation of state which would be another process that could produce density fluctuations. This talk explores topological defects, yet another mechanism that generates density fluctuations in an initial smooth universe through equation of state fluctuations.

\section{TOPOLOGICAL DEFECTS}

Topological defects can arise at a symmetry breaking in the early universe. Symmetry breaking is a fundamental part of modern particle physics and most of us suspect that the standard model of particle physics, with broken $S U(3) \times$ $S U(2) \times U(1)$ symmetry, is much simpler at higher energies. This suggests that the universe underwent a series of phase transitions and different regions of space may be in different degenerate vacuum states. As Kibble [2] noted, different causal disconnected regions of space will settle into different vacuum states. These field misalignments can generate topological defects and can generate density fluctuations that could have seeded galaxies [3, 4] 
The type of defect that forms at a phase transition depends on the homotopy group of the vacuum manifold after the symmetry breaking. This can be seen by considering a simple potential for a N-dimensional scalar field, $\vec{\phi}$ :

$V(\phi)=\lambda\left(\phi^{2}-\phi_{0}^{2}\right)^{2}$

If $\phi$ is a one dimensional field, then different regions of space will settle into one of the two vacuum state, $\phi=\phi_{0}$ and $\phi=-\phi_{0}$. Domain Walls will seperate these two vacuum states. If $\phi$ is a two dimensional field, then the vacuum manifold is $\phi=\phi_{0} \exp (i \theta)$, where the phase angle $\theta$ varies over space. Strings will form along lines at which $\oint \nabla \theta d \vec{s} \neq 0$. Monopoles, textures and nontopological textures are associated with higher dimensional vacuum manifolds (see table 1 ).

The cosmological behavior of a defect will depend upon whether the symmetry is gauged or global. While gauged cosmic strings and global strings have very similar cosmological behavior, this is not true of the other defects. Gauged monopoles are cosmologically dangerous - they interact so weakly that once formed, they do not annihilate and can easily dominate the energy density of the universe. Global monopoles, on the other hand, have significant long range interaction. They annihilate quickly enough so that their energy density is a constant fraction of the closure density and are potentially interesting source of density fluctuations 6.5. Gauged textures do not produce significant density flucutations, while global texture are similar to monopoles and are a potentially interesting source of density fluctuations |7].

\subsection{Evolution of Defects}

Strings, global monopoles and textures in a flat universe have a relatively simple dynamical evolution. As the universe expands, the defect network untangles and the characteristic scale over which the field changes significantly, the coherence scale of the field, grows linearly with conformal time. Numerical simulations of cosmic strings find that the curvature scale of the strings is a constant fraction of the horizon size 8 [10]. In numerical simulations of global field evolution in an expanding universe, the characteristic scale of textures and the spacing between textures is also found to grow linearly with conformal time in a radiation and matter-dominated universe 11, 12 This scaling form has been used in many studies of these models 13 16] to estimate the density fluctuations produced by these defects. This scaling form implies that the energy density in the defects is a constant fraction of the closure density. If the scale of symmetry breaking is $\phi_{0}$, then the energy density in defects is $\delta \rho \sim \dot{\phi}^{2} \sim\left(\phi_{0} / \eta\right)^{2}$, where $\eta$ is the age of the universe measured in conformal time, and all our units are $\hbar=c=1$. Since the closure density is $\rho \sim\left(M_{P l} / \eta\right)^{2}$, this implies that the energy density in defects is scale invariant,

$\frac{\delta \rho_{\text {defect }}}{\rho} \simeq\left(\frac{\phi_{0}}{M_{\text {Planck }}}\right)^{2}$

Note that the energy density in defects is constant. As the amplitude of density fluctuations produced by defects is of order their energy density, the constant energy density fraction in global field theories leads to a scale-invariant spectrum of density fluctuations [3], similar to that predicted by inflation. The amplitude of CBR fluctuations detected by COBE 14 and the amplitude of galaxy fluctuations imply that $\delta \rho / \rho \sim$ $10^{-4}-10^{-5}$. Thus, if defects are the source of density fluctuations, then $\phi_{0} \sim 10^{-2} M_{\text {Planck }}$ suggesting a GUT scale phase transition.

\subsection{Spectrum of CBR Fluctuations}

On the large-angular scales probed by COBE, the dominant source of microwave background fluctuations in most models are metric fluctuations 17. These fluctuations can be divided into three classes, scalar fluctuations, vector fluctuations and tensor fluctuations 18, 19. The growing and decaying matter modes are scalar fluctuations. The vector fluctuations, vorticity fluctuations, are subdominant in defect models and not predicted in inflationary models. The tensor modes are the gravity wave fluctuations that have attracted significant attention in the past year in the context of inflationary models.

In defect models, the dominant source of microwave fluctuations are scalar fluctuations. Thus, we can get a rough physical understand- 
Table 1

Types of Defects

\begin{tabular}{|c|c|c|c|c|}
\hline Defect & $\mathrm{N}$ & Homotopy Group & Global & Gauged \\
\hline$\overline{\text { Domain Walls }}$ & 1 & $\overline{\Pi_{0}}$ & $\mathrm{x}$ & \\
\hline Strings & 2 & $\Pi_{1}$ & $\mathrm{x}$ & $\mathrm{x}$ \\
\hline Monopoles & 3 & $\Pi_{2}$ & $\mathrm{x}$ & \\
\hline Textures & 4 & $\Pi_{3}$ & $\mathrm{x}$ & \\
\hline N.T. Textures & $N>5$ & $\Pi_{4}, \Pi_{5}, \ldots$ & $\mathrm{x}$ & \\
\hline
\end{tabular}

ing for the predictions of these models by focusing only on fluctuations in the gauge-invariant Bardeen scalar potential $\Phi$. On scales smaller than the horizon size, this potential is the familar Newtonian gravitational potential. Fluctuations in the gravitational potential contribute to the CBR fluctuations through two terms [17,20]:

$$
\left.\frac{\delta T}{T}\left(\eta_{0}\right) \simeq \frac{\Phi}{3}\right|_{\eta_{L S}} ^{\eta_{0}}+\left.2 \int_{\eta_{L S}}^{\eta_{0}} \frac{\partial \Phi}{\partial t}\right|_{x=x(t)} d t+\frac{\delta T}{T}\left(\eta_{L S}\right)(3)
$$

The first term describes fluctuations at the surface of last scatter. The second term describes microwave fluctuations produced as the photons travel from the surface of last scatter to the observer. $\eta_{L S}$ is the conformal time at the surface of last scatter and $\eta_{0}$ is the conformal time today. The third term describes contributions to the CBR due to variations in the photon/baryon or photon/dark matter ratio. In inflationary models in a flat universe, only the first term is nonzero, thus, the CBR fluctuations are probe potential fluctuations at the surface of last scatter. This has led to the oversimplifying statement that COBE is probing density fluctuations at $z=1000$. This need not be true. In defect models and all models in open or $\lambda$-dominated universes, $\dot{\Phi}$ is non-zero. In most of these models, the quadrupole is produced primarily by the decay of potential fluctutations at $z \sim 1 / \Omega$. In the PIB model, the third term is an additional source of CBR fluctuations.

Since defects tend to generate potential fluctuations predominantly on their coherence scale, they predominantly produce microwave fluctuations on angular scales close to that subtended by the horizon at a given redshift. In a flat universe, $\theta_{H} \simeq(1+z)^{-1 / 2}$ radians. Thus, textures collapsing at $z \sim 50$ are the dominant source of CBR fluctuations on angular scales around $10^{\circ}$. Since defects produce a scale invariant spectrum of potential fluctuations below the coherence scale, they also produce a scale invariant spectrum of fluctuations in the microwave sky. Thus, like inflation, they predict that the amplitude of the harmonic multipole, $c_{l}$, scales as $l^{-2}$ at large $l$. However, at scales larger than the coherence scale today, microwave fluctuations in the defects models are suppressed and $c_{l} \propto l^{-1}$ rather than $\propto l^{-2}$ at small $l$. As we will see when we consider open universe models, this will lead to a dramatic suppression of the quadrupole and other low multipole moments.

\subsection{Spectrum of Density Fluctuations}

Since defect evolution is a scale-invariant process, defects produce a density fluctuation spectrum similar to that predicted in the simplest inflationary models. As noted earlier, defects tend to produce fluctuations primarily at their coherence scale at a given epoch. Since density fluctuations basically do not start growing until the universe is matter dominated, the power spectrum of density fluctuations is peaked at the wavenumber associated with the coherence scale at equality. On scales larger than the coherence scale at equality and smaller than the coherence scale today, the spectrum scales as $\propto k$ as the process of generating fluctuations is scale-invariant over this region. On scales larger than the coherence scale today, the density spectrum scales as $k^{4}$ as there has not yet been time for any causal process to produce density fluctuations on these large scales. Defects collapsing in the future will produce fluctuations on these scales.

On scales smaller than the coherence scale at matter-radiation equality, the shape of the den- 
sity spectrum is determined by the nature of the dark matter. Thus, over the range of scales probed by observations of large-scale structure, the shape of the power spectrum in a CDMdominated defect model is similar to that in a CDM-dominated inflationary model. However, in the defect model, the peak of the power spectrum occurs at a somewhat smaller scale as the field coherence scale is smaller than the horizon size in these models. This reduction of large scale power is particularly dramatic in a string-seeded $\Omega=1$ models 14. As we will see, this loss of large-scale power is likely to be deadly for these models.

\subsection{Numerical Simulations of Defects in Flat Universe}

While the rough physical arguments of the previous section yield a qualititative understanding of the predictions of defects models, quantitative comparision with observations requires largescale numerical simulations. While I will focus on results from simulations described in detail in [22], the basic approach is similar for all defect simulations 21, 23, 14.

The first part of the simulation is the evolution of the defect in the expanding universe. There are two basic approaches to field evolution, either the Lagrangian of the fundamental theory is used to determine the field evolution equation in the expanding universe 21] or the field is evolved as a free field with the constraint that $|\vec{\phi}|=\phi_{0}\left[\begin{array}{l}7,23 \\ \text {, }\end{array}\right.$ 22]. Both approaches yield similar results.

Contrary to what one might naively expect, the complexity of the numerical simulation actually decreases with increasing $N$. For $N \longrightarrow \infty$, the field evolution can be solved exactly [24], and only has small departures from Gaussianity at $N>6$ [25]. At $N=4$ and below, real defects exist in space time, causing singular field configurations and the associated numerical uncertainties. For textures, the problem only arises at a small number of events in space time which we model with the "spin flipping" technique22, but for global monopoles the singularity is real and needs to be smeared out over a few grid cells. For strings, the situation is much more difficult, because global strings carry an energy density proportional to $\log \left(r_{c} / r_{\xi}\right)$ the $\log$ of the core radius (a GUT scale) to the mean string separation (a fraction of today's visible universe). This ratio is nearly constant, while in the simulation we do not have nearly enough dynamic range.

The field evolution determines the stress energy tensor of the defects, which serves as a source for the generation of metric fluctuations. In the numerical simulations, all three types of metric fluctuations (scalar, vector and tensor) are computed. By evolving the scalar fluctuations to today, we can compute the predicted amplitude of density fluctuations in a given model. The other metric fluctuations terms are needed to compute $\delta T / T$.

Photons are propogated along geodesics towards an observer placed somewhere in the simulation. As the photon moves towards the observer, the metric fluctuations produce temperature fluctuations that are summed along the photon path. In recent simulations, Coulson et al. 26] have also included the effects of photon scattering off of free electrons, this scattering damps fluctuations on angular scales smaller than the horizon size at the surface of last scatter. At the end of a simulation, the microwave sky map can be compared directly to the COBE observations. The COBE detection of fluctuations on the $10^{\circ}$ scale is used to normalize the thoeries and to compare the theory predictions to observations of largescale structure.

The multipole spectrum of CBR fluctuations found in the numerical simulations is consistent with our expectations for a curvature theory. On scales smaller than $\sim 60^{\circ}$, the angular scale corresponding to the coherence scale today, the CBR fluctuations are scale invariant. This implies that $c_{l} l(l+1)$ is constant. On the largest angular scales, there is a weak suppression of the dipole, quadrupole and octopole. However, these would be difficult to detect given the cosmic variance. The predicted spectral shape, similar to that predicted by inflationary scenarios, is consistent with the current observations. While the fluctuations predicted for COBE are mildly non-Gaussian, the current observations can not distinguish this signature. High signal-to-noise measurements on small angular scale will be needed to detect this signature of topological defects 26. 


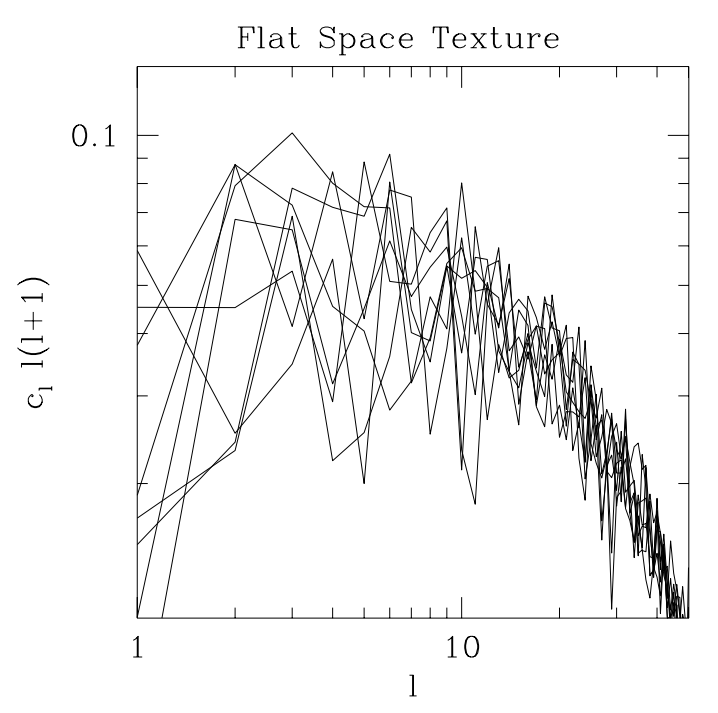

Figure 1. Fluctuations from Global Texture in an $\Omega=1$ universe

This figure from Pen et al. (1994) shows the calculated CBR multipole spectra in a global texture simulation in a flat universe. The different lines correspond to the CBR fluctuations seen by different observers at various locations in the simulation box. The dispersion in their multipole measurements in a signature of the expected cosmic variance.

\subsection{Comparison with Observations}

Unfortunately, the results for most defect theories in a flat universe are quantitatively disappointing. These models in a flat CDM-dominated universe normalized to COBE predict low amplitudes of mass fluctuations on the $8 / \mathrm{h} \mathrm{Mpc}$ scale of $0.25 h_{50}^{-1}$ [22]; these large bias factors is somewhat concerning. Even more concerning for the theory is that it underpredicts the amplitude of mass fluctuations on the $20 / \mathrm{h} \mathrm{Mpc} \mathrm{scale} \mathrm{by}$ a factor of 6 ! Albrecht and Stebbins 14 reach similar conclusions for cosmic string models in a CDM-dominated universe. This model also underpredicts the amplitude of mass fluctuations at the $20 / \mathrm{h}$ Mpc scale and appears unlikely to be consistent with the APM 31], IRAS 32 and CfA 33] surveys. Because of the small coherence length of cosmic string theories, their power spectra peak at smaller scales than "standard" inflationary models, thus, we suspect that more detailed analysis of the strings + HDM scenario will also conclude that it does not predict large enough fluctuations at scale $>20 / \mathrm{h} \mathrm{Mpc}$. All of these discrepancies, of course, rely on numerical simulations. It is certainly possible that more detailed simulations will remove the discrepancies between theory and observations. They may also exacerbate the discrepancies.

Defect theories are not alone in having difficulties fitting the observed galaxy distribution. Most inflationary scenarios can not simulateously fit the observed level of COBE fluctuations and the small pair-wise velocity dispersions observed at small scales. This has lead large-scale structure simulators into their baroque phase as once simple theories acquire additional free parameters (e.g., mixed dark matter, cosmological constants, tensor modes, "designer" spectra).

We believe that these discrepancies should encourage us to reconsider our assumptions and explore open universe models. There are a host of observations that point towards an open universe and as we will see these models are remarkably successful at explaining the observed large scale structure.

\section{OBSERVATIONAL EVIDENCE FOR LOW $\Omega$}

Observations of the dynamics of clusters, their gas content and the age problem all provide strong motivation for considering open models.

There are several independent techniques that can be used to determine the mass-to-light ratios of clusters: measurements of the velocity dispersion of galaxies, observations of X-ray temperature profiles and modelling of the observed pattern of gravitational lensing of background objects by a cluster. All of these techniques yield similar estimates for the mass of clusters and imply that if mass traces light on the scales of clusters, $\Omega$ is $\sim 0.2$ 27] For example, detailed studies 
of the dynamics of the Coma cluster finds an M/L of $310 \pm 50 h$ on the scale of $5 / h \mathrm{Mpc} 28$. If this large region is a fair sample of the universe, then $\Omega \simeq 0.2$.

Measurements of the ratio of the mass of hot gas in clusters to the total mass of a cluster imply that baryons comprise at least $1 / 6$ of the mass of the cluster for $H_{0}=50 \mathrm{~km} / \mathrm{s} / \mathrm{Mpc} 29$. Combining this with estimates of the baryon density from from big bang nucleosynthesis 30] $\Omega_{b} h^{2} \simeq 0.015$, suggests that $\Omega<<1$. These observations provide further evidence that the universe is open or that the baryons are not very good tracers of the mass on the scale of clusters of galaxies.

Observations of the galaxy motions also provide evidence that $\Omega<<1$. On scales of $\sim 1 \mathrm{Mpc}$, the measured pairwise velocities of galaxies, $\sim$ $300 \mathrm{~km} / \mathrm{s}$, implies that $\Omega \simeq 0.15 \exp ( \pm 0.4)$ [34]. Numerical simulations of CDM models normalized to COBE predict a pairwise dispersion of $1000 \Omega^{0.6} \mathrm{~km} / \mathrm{s}$. The thinness of the structure in the observed large-scale structure such as the "Great Wall" 35] the caustic structure seen in superclusters[36] as well as the cold local Hubble flow inferred from observations of galaxy peculiar velocities 37, provide additional evidence that the large small-scale random velocities predicted in an unbiased $\Omega=1$ model are not observed.

Observations of galaxy motions on large scales do, however, hint at larger values of $\Omega$. While analysis of the redshift structure of the IRAS galaxy sample of galaxies imply that $\Omega^{0.6} / b_{I R A S} \sim 0.540$ larger densities are suggested by the comparison of the galaxy velocities with the distribution of IRAS galaxies using the POTENT algorithm 38]: $\Omega^{0.6} / b_{I R A S} \sim$ $1.28_{-0.59}^{+0.75}$, where $b_{I R A S}$ is the ratio of the fluctuations in the IRAS selected galaxies to the ratio of the fluctuations in the underlying mass distribution. The POTENT estimate is subject to uncertainities due to the non-linear Malmquist biases [39] Since the IRAS sample is known to be biased against elliptical galaxies, it is quite plausible that optical galaxies are better tracers of the underlying mass distribution than infrared galaxies. Thus, if we use the observed ratio of optical to infrared galaxy fluctuations 41, $b_{\text {optical }} / b_{\text {IRAS }}=1.7$ even these observations suggest $\Omega<1$ and are compatible with $\Omega \sim 0.2$.

Requiring that the age of the universe is older than that of stars in globular clusters provides additional evidence that $\Omega<<1$. Most recent measurements of the Hubble constant suggest that $H_{0} \sim 75 \mathrm{~km} / \mathrm{s} / \mathrm{Mpc} 42$. On the other hand, the age of the universe, $t_{0}$, should exceed 13 billion years, the lower limit estimated for the age of globular clusters 43. These results suggest that $H_{0} t_{0} \geq 0.8$. This is in excess of the predictions of a flat $(\Omega=1)$ matter dominated universe, $H_{0} t_{0}=2 / 3$, and is more consistent with either an open universe, or a universe whose energy density is dominated by a cosmological constant.

These arguments suggest that there is strong astronomical motivation for considering model in which $\Omega<1$. This need not imply that the universe is open. If the universe is dominated by a cosmological constant, then the universe could still be flat. It is difficult, however, to understand the origin of such a small cosmological constant, $\Lambda \simeq 10^{-128} M_{\text {Planck. }}^{4}$. Thus, we will focus on open models with $\Lambda=0$.

\section{DEFECTS IN AN OPEN UNIVERSE}

\subsection{Analytical Model}

As a first step towards exploring the evolution and effects of defects in an open universe model, a simple analytical model provides a useful estimate the density fluctuations produced by defects 16]. Comparision with numerical simulations (see figure 2) suggest that this model may be an accurate description of the density fluctuations produced by defects.

Since defects generate density fluctuations through variations in the equation of state, the gravitational effects of defects can be approximated by describing the evolution of the pressure fluctuations produced by defects, $\delta p$. On scales larger than the defect coherence scale, $l_{c o h}$, the pressure fluctuations produced by the defects are uncorrelated. Thus, for small $k, \delta p$ is independent of $k$. On scales smaller than the coherence scale, $\delta p$ is suppressed. Thus, defect effects can be described by,

$\delta p(k)=\Theta(\beta k \eta-1)$ 


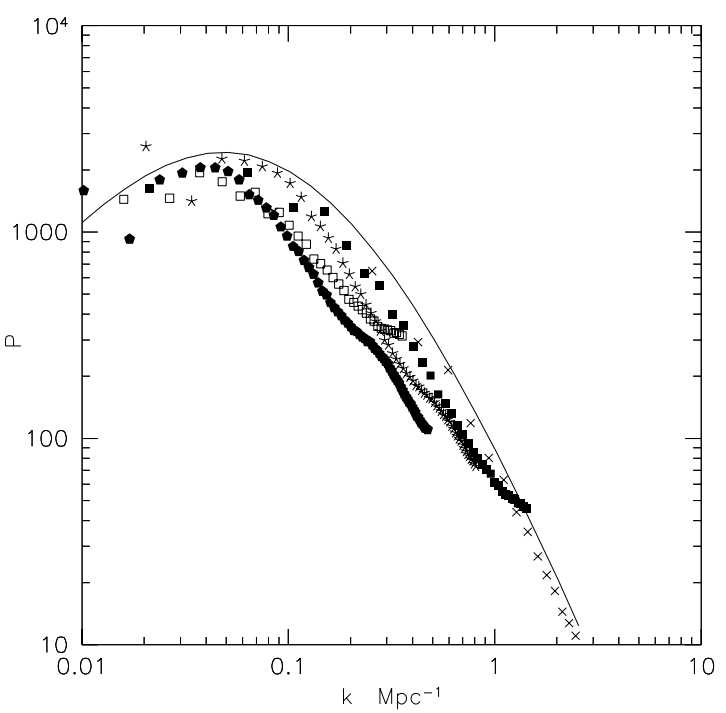

Figure 2. Density Fluctuations (Analytical Model)

This figure from Spergel(1993) compares the density fluctuation spectrum predicted by an analytical model for the gravitational effects of defects with numerical simulations (Pen et al. 1994). The solid line is the predictions of analytical theory and the triangles and squares are the results of various simulations with different grid sizes.

where $\beta$ is the ratio of the defect coherence scale to the horizon size. For texture models, $\beta \sim 1 / 3$, while for string models, $\beta \sim 1 / 5-1 / 10$. Once we have described the evolution of pressure fluctuations, the gauge-invariant approach of Kodama and Sasaki 19 can then be used to compute the evolution of the gravitational potential and density fluctuations. A similar approach has been used by Albrecht and Stebbins 14 and Perivoropolous 15] to model the generation of density fluctuations by cosmic strings.

This analytical model suggest that defects are extremely promising. The spectrum is similar to that reported based on the reanalysis of the COBE data 48 and the COBE normalized theory implies reasonable galaxy density fluctuations, $\sigma_{8} \sim 0.5 \pm 0.2$ for $\Omega=0.2$ and $h=0.8$. This is consistent with determinations of $\sigma_{8}$ from the cluster mass function: $0.6<\sigma_{8}<0.8$. This success of the analytical model suggests that defects in an open universe merit further investigation.

\subsection{Numerical Simulations of Defects in an Open Universe}

On scales much smaller than the curvature scale, the same numerical techniques that have been used to simulate flat universe models can be easily rescaled to simulate open universe models. However, on the large scales probed by COBE, significant changes must be made in any algorithm in order to simulate the evolution of potential fluctuations in negatively curved space. We have developed codes for simulating defects in the non-Euclidean negatively curved open universe.

We found that a convenient approach to evolving potential fluctuations and microwave fluctuations in an open universe was to use the Poincaré metric:

$d s^{2}=\frac{d x^{2}+d y^{2}+d z^{2}}{z^{2}} \quad z>0$

This metric has significant advantages over the more familiar Robertson-Walker metric as it does not have a prefered position for the observer and geodesics in this metric are simple analytical expressions for all positions in space 45. It is straightforward to lay down evenly spaced grid points and it is possible to make the grid periodic in $\mathrm{x}$ and $\mathrm{y}$. This semi-periodic grid is the three dimensional analogue to the surface of rotation generated by a tractrix. In the $\mathrm{z}$ direction, we extend the grid beyond the horizon of the observers and use mirror boundary conditions. While this is mildly wasteful of memory, it has the advantage of simplicity.

Texture, non-topological texture and monopole theories can be accurately described on cosmological scales by a non-linear sigma model[ [7]. We use this model to evolve these theories using the algorithms described in Pen et al.22]. At the beginning of the simulations, when $\Omega \simeq 1$, the field in the open universe simulations has approximately the same scaling energy density as in the 
flat universe simulations. As the universe begins to become open and expand more quickly, the field oscillations are damped by Hubble expansion and the scaling energy density drops. In a flat universe, the scaling density implies $\dot{\phi}^{2} \propto(\nabla \phi)^{2} \propto 1 / \eta^{2}$. When $\Omega=0.2$, the kinetic energy density has dropped to $70 \%$ of its flat universe value. This lower energy density will lead to a suppression of CBR fluctuations on large angular scales. A decreased kinetic energy implies an increased gradient energy, but since the integral is over $\dot{\Phi}$, it is only the time dependent part which contributes to the Sachs-Wolfe effect.

In our open universe simulations, we focus on the scalar fluctuations produced by isotropic stresses. In our flat universe simulations, we found that these scalar fluctuations were the dominant source of CBR fluctuations. In the open universe calculations, we will assume that the ratio of tensor and vector fluctuations to scalar fluctuations is the same as in the flat universe calculations and use this to rescale our CBR fluctuations. Since the textures and monopoles move somewhat slower in an open universe, this should suppress tensor and vector modes, thus, our rescaled CBR fluctuations result may be slightly overestimated. Throughout our open universe calculations, we use the gauge-invariant formalism described in 119 to calculate the potential fluctuations produced by the isotropic stresses.

In our calculations, we compute these fluctuations by following photons as they propogate towards an observer in the center of the box and compute the Sachs-Wolfe contribution to $\delta T / T$ (equation 3). We then use a spherical harmonic expansion to compute the amplitude of each multipole moment. The results in figure 3 are for several different observers in the same box in an $\Omega=0.2$ texture-seeded universe. The figure shows the dramatic signature predicted by open universe models: a suppression of low multipoles.

The relationship between angular scale and horizon size at a given redshift, $\theta \simeq \Omega(1+z)^{-1 / 2}$, leads to a characteristic signature for open universe models with defects on the large angular scales probed by COBE. This effect is more dramatic than in open universe models that enter the FRW phase with curvature fluctuations 46 .

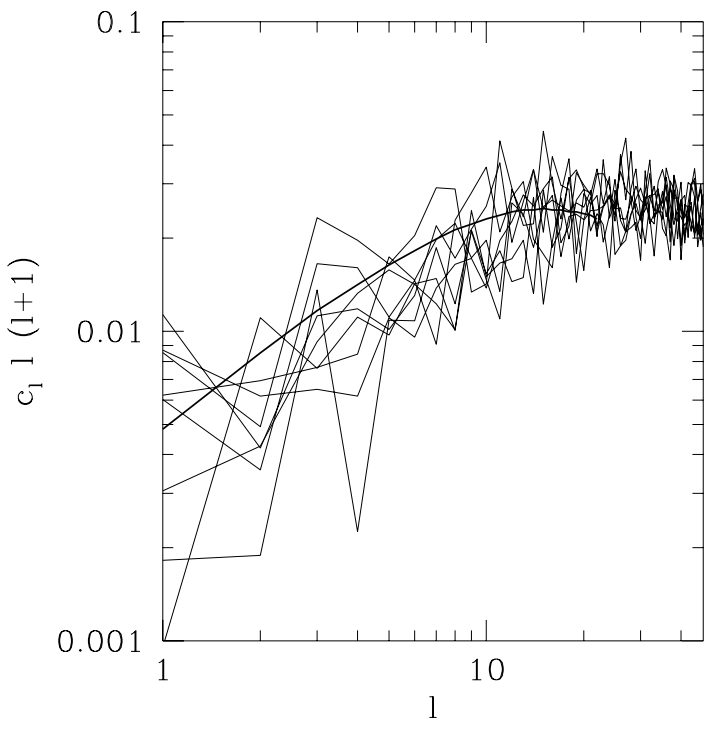

Figure 3.

The different solid curves trace the multipole spectrum observed by different observers in a open $\Omega=0.2$ CDM dominated universe. The temperature fluctuations are normalized so that $8 \pi^{2} G \phi_{0}^{2}=1$ with the tensor and vector multipole contributions scaled from the flat universe model. The heavy line is the prediction of the analytic model 16 .

In a flat universe, regardless of whether fluctuations are seeded by defects or by inflation, the multipole fluctuations are expected to scale as $c_{l} \propto 1 /[l(l+1)]$ for $l \lesssim 50$. Open models follow this form for $l \gtrsim 20$ as curvature was relatively unimportant when fluctuations were generated on these scales. However, on large angular scales, these models predict a suppression of CBR fluctuations. This suppression of low multipoles contrasts with inflationary models that predict an enhancement of low multipoles due to gravitational waves 47.

The current observational results shown are tantalizing but not yet definitive. The COBE data 48 suggest that the low multipole moments are suppressed, a trend that appears to be con- 
firmed by the larger amplitude fluctuations seen on $l \sim 20$ by the MIT experiment 49 and $l \sim 30$ by the Tenerife experiment 50 . There remain large uncertainties in these measurements and further observational work will be needed before any strong statement can be made about the multipole spectrum. It is, however, very intriguing that open universe models make clear predictions at low $l$ and that the data seems to follow this trend.

Microwave predictions on smaller angular scales depend on the ionization history of the universe. Without reionization, low $\Omega$ models with scale-invariant spectra are not consistent with microwave limits on small angular scales 51. If an early burst of star formation reionized the universe, then microwave fluctuations are suppressed on angular scales smaller than that corresponding to the horizon scale at the redshift at which the universe was last optically thick. In a flat universe with $H_{0}=50 \mathrm{~km} / \mathrm{s} / \mathrm{Mpc}$ and $\Omega_{b}=0.06$, the surface of last scatter, $\tau=2 / 3$ corresponds to $z=60$ and microwave fluctuations are suppressed on scales smaller than $\sim z_{L S}^{-1 / 2} \sim 6^{o}$, thus, these models predict that fluctuations are suppressed for $l>3047$. This early reionization appears to be inconsistent with several of the reported detections of microwave fluctuations 52, 53, 55, 54 on scales of $\sim 1^{\circ}$. In an open $\Omega=0.2$ universe with the same parameters, the surface of last scatter corresponds to $z=75$, and microwave fluctuations are only suppressed below $\Omega_{0} z_{L S}^{-1 / 2} \sim 1^{o}$. This would be consistent with both the detections at the $30^{\prime}-2^{\circ}$ scale and the limits of $\delta T / T<2.3 \times 10^{-5}$ recently obtained on angular scales of 12 '56]. As the observations rapidly improve, we will hopefully be able to detect (or rule out) the distinctive CBR signature of open universe models.

\subsection{Density Fluctuations in an Open Uni- verse}

The COBE observations can again be used to normalize the one free parameter in defect theories for fixed $\Omega$ and $h$ : the scale of symmetry breaking, $\phi_{0}$. With this parameter fixed, we can determine the predicted galaxy power spectrum by scaling the results of our earlier simulations of defects in a flat universe. This calculation ignores the effects of baryons which will slightly suppress the spectrum at scales smaller than the Silk damping scale.

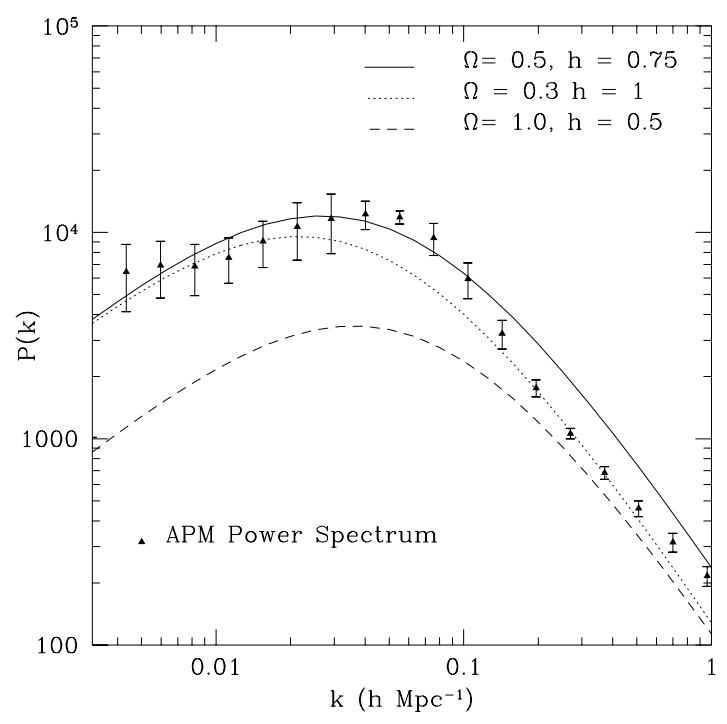

Figure 4. Comparision of COBE normalized theories to APM Galaxy Survey

The open squares are the power spectrum of galaxy fluctuations computed from the APM survey. The lines show the predicted power spectrum for COBE-normalized defect models with different values of $\Omega$ and $h$. The bias is assumed to be 3 for the $\Omega=1$ and 2.5 for the $\Omega=0.3$ and $\Omega=0.5$ models. These bias factors have a " $1 \sigma$ " uncertainty of $20 \%$.

The agreement between the texture-seeded open model and the APM observations is remarkable. The open model appears to fit both the shape of the CBR spectrum as determined from the COBE, MIT and Tenerife experiments and the shape of the galaxy power spectrum.

While our numerical simulations of defects in an open universe have focused on textures, the basic conclusions are likely to be equally valid 
for strings, global monopoles and non-topological texture. Strings with their much smaller coherence scale should predict an even larger suppression of low multipoles and a "break" in the spectrum at large $l$ for fixed $\Omega$. In a flat universe dominated by cold dark matter, the cosmic string power spectrum is not a good fit to the observed galaxy distribution 14, however, the same model rescaled to an open universe does remarkably well at fitting the observations. The required string tension, $G \mu \simeq 3-5 \times 10^{-5}$ is roughly consistent with the current estimate of string induced CBR fluctuations [26,58, 22]. In an open universe, the millisecond pulsar constraint on the energy density in gravity waves implies a much weaker constraint on $G \mu$, thus, these limits which are restrictive for $\Omega=1$ are less troublesome for the model in an open universe. We suggest that open universe string model deserves more careful consideration.

Another intriguing model is a low $\Omega$ baryonsonly string-seeded model. In this model, string wakes would be the dominant mechanism for seeding structure 59,60]. We plan to investigate this model in more detail. Some further analyses and speculations are reported in 61.

\section{CONCLUSIONS}

The generation of density fluctuations by defects is a plausible alternative to the generation of fluctuations by quantum fluctuations during inflation. Defects in an open universe are a particularly exciting model as texture in an open CDM dominated model appears to be consistent with the observed CBR spectrum and the observed level of galaxy fluctuations.

\section{REFERENCES}

1. Peebles, P.J.E. ApJ, 315 (1987) L73.

2. Kibble, T.W.B. J. Phys, A9 (1976) 1387.

3. Press, W.H., Physica Scripta, 21 (1980) 702.

4. Vilenkin, A., Physics Reports, 121 (1985) 263.

5. Barriol, M. and Vilenkin, A., PRL, 63 (1989) 341.
6. Bennett, D.P. and Rhie, S.H., PRL 65 (1990), 1709.

7. Turok, N., PRL, 63 (1989) 2625.

8. Bennett, D.P. and Bouchet, F.R. PRD 43 (1991) 2733.

9. Albrecht, A. and Turok, N., PRD 40 (1989) 973.

10. Allen, B. and Shellard, E.P.S., PRD 45 (1992) 1898.

11. Spergel, D.N., Turok, N., Press, W.H. and Ryden, B.S. PRD 43 (1991) 1038.

12. Nagasawa, M. and Sato, K., Int. J. Mod. Phys. D1 (1992) 427.

13. Gooding, A., Park, C., Spergel, D., Turok, N. and Gott, J.R. ApJ 393 (1992) 42.

14. Albrecht, A. and Stebbins, A. PRL, 68 (1992) 2121.

15. Perivolaropoulos, L., Phys. Lett. B298 (1993) 305.

16. Spergel, D.N. ApJ(Letters) 412 (1993) L5.

17. Sachs, K. and Wolfe, A.M., ApJ 147 (1967) 73.

18. Bardeen, J.M., PRD, 22 (1980) 1992.

19. Kodama, H. and Sasaki, M. Prog. Theor Phys. Suppl., 78 (1984) 1.

20. Gouda, N. and Sugiyama, N. ApJ 395 (1992) L59.

21. Press, W.H., Ryden, B.S., and Spergel, D.N. ApJ 347 (1992) 590.

22. Pen, U., Spergel, D.N. and Turok, N. PRD (1994) in press.

23. Bennett, D.P. and Rhie, S. ApJ, 406 (1993) L7.

24. Turok, N. and Spergel, D.N., PRL 66 (1991) 3093.

25. Jaffe, Andrew H., FERMILAB no. 93/317-A (1993).

26. Coulson, D., Ferreira, P., Graham, P. and Turok, N., Princeton University Physics preprint PUP-TH-93/1429 (1993).

27. Peebles, P.J.E. Principles of Physical Cosmology, (Princeton: Princeton University Press) (1993).

28. Hughes, J.P. ApJ 337, (1989) 21.

29. White, S.D.M., in "Clusters and Superclusters of Galaxies", A.C. Fabian (ed.) (Kluwer Academic Publishers: Netherlands), (1992) 17. 
30. Walker, T.P., Steigman, G., Kang, H., Schramm, D.N., Olive, K.A. ApJ 376 (1991) 51.

31. Baugh, C.M. and Efstathiou, G. MNRAS 265 (1993) 145.

32. Fisher, K.B., Davis, M., Strauss, M.A., Yahil, A. and Huchra, J.P. ApJ 402 (1993) 43.

33. Vogeley, M.S., Park, C., Geller, M.J., Huchra, J.P., ApJ, 391 (1992) L5.

34. Davis, M. and Peebles, P.J.E ApJ, 267, (1983) 465.

35. Geller, M.J. and Huchra, J.P., Science 246, (1989) 897.

36. Regos, E. and Geller, M.J. AJ, 98, (1989) 735.

37. Ostriker, J.P. and Suto, Y. ApJ 348, (1990) 378.

38. Dekel, A., Bertschinger, E., Yahil, A. Strauss, M.A., Davis, M. and Huchra, J.P. ApJ 412,(1993) 1.

39. Gould, A. ApJ 412, (1993) L55.

40. Fisher, K.B., Davis, M., Strauss, M.A., Yahil, A. and Huchra, J.P. IAS preprint (1993).

41. Lahav, O., Nemirroff, R.J. and Piran, T. ApJ 350, (1990) 119.

42. Jacoby, G.H. et al. PASP 104, (1992) 599.

43. Deliyannis, C.P., Demarque, P. and Pinsonneault, M.H., ApJ 347, (1989) L73.

44. Wright, E.L. et al., Ap. J. (Letters), 396, (1992) L13.

45. Wilson, M.L. ApJ 273, (1983) 2.

46. Kamionkowski, M. and Spergel, D.N., submitted to ApJ (1993).

47. Bond, J.R., et al., CITA preprint (1993).

48. Wright, E.L., results presented at the Capri CMBR workshop, September 1993.

49. Ganga, K. Cheng, E., Meyer, S. and Page, L., Texas/PASCOS 92: Relativistic Astrophysics and Particle Cosmology, ed. C.W. Akerlof and M.A. Srednicki, (1993) p. 795.

50. Watson, R.A., et al., Nature, 357, (1992) 660.

51. Bardeen, J.M, Bond, J.R. \& Efstathiou, G., ApJ, 321 (1987) 28.

52. Devlin, et al. Texas/PASCOS 92: Relativistic Astrophysics and Particle Cosmology, ed. C.W. Akerlof and M.A. Srednicki, (1993) p. 809

53. Wollack, E.J.; Jarosik, N.C.; Netterfield, C.B.; Page, L.A.; Wilkinson, D. "A mea- surement of the anisotropy in the cosmic microwave background radiation at degree angular scales", Princeton University Physics Department preprint (1993).

54. Cheng, E.S.; Cottingham, D.A.; Fixsen, D.J.; Inman, C.A.; Kowitt, M.S. "A measurement of the medium-scale anisotropy in the cosmic microwave background radiation", Goddard preprint (1993).

55. Dragovan, M., talk presented at Princeton University Physics department (1993).

56. Tucker, G.S., Griffin, G.S., Nguyen, H. and Peterson, J.B., Princeton University Physics Department preprint (1993).

57. Pen, U. and Spergel, D.N., in preperation.

58. Bennett, D.P., Stebbins, A. and Bouchet, F.R., ApJ (Letters), 399 (1992) L5.

59. Vachaspati,T. PRL 57 (1986) 1655.

60. Melott, A.L. and Scherrer, R.J. ApJ 331 (1988) 38 .

61. Spergel, D.N. and Pen, U., submitted to Nature. 